Gutembergue Livramento

Tânia Franco ${ }^{2}$

Alaíde Livramento ${ }^{3}$

\title{
A ginástica terapêutica e preventiva chinesa Lian Gong/Qi Gong como um dos instrumentos na prevenção e reabilitação da LER/DORT
}

\author{
Lian Gong/Qi Gong - Chinese therapeutic and preventive \\ exercise as an instrument for Cumulative Trauma Disorder \\ prevention and rehabilitation
}

\begin{abstract}
${ }^{1}$ Professor da graduação e mestrando da Escola Bahiana de Medicina e Saúde Pública (EBMSP); Mestre de Kung Fu, Tai Chi Chuan e Qi Gong pelo Centro de Estudos e Pesquisas de Shenzhen, GuangDong, China; Fisioterapeuta com especialização em Fisiologia do Exercício; formação em Engenharia e Biologia; Presidente do Instituto Brasileiro de Ensino e Pesquisa em Qi Gong e Medicina Chinesa (Ibrapeq).

2 Pesquisadora do CRH/UFBA, com formação em Economia, Medicina, Mestrado e Doutorado em Ciências Sociais; formação em Qi Gong.

${ }_{3}^{3}$ Professora de Qi Gong e Tai Chi Chuan Instituto Brasileiro de Ensino e Pesquisa em Qi Gong e Medicina Chinesa (Ibrapeq).
\end{abstract}

Contato:

Gutembergue Livramento

Rua Renato de Menezes Berenguer, 185, Apto. 802B, Pituba. Salvador, Bahia, Brasil.

CEP: 41830-315

E-mail:

gutemberguelivramento@yahoo.com.br

\section{Resumo}

Este ensaio traz elementos da medicina ocidental, da medicina tradicional chinesa e da sociologia do trabalho para reflexão sobre o Qi Gong - exercícios terapêuticos, um dos pilares da Medicina Tradicional Chinesa (MTC) - como importante meio de prevenção da LER/DORT e recuperação da saúde. Considerando a natureza interdisciplinar e diversos níveis de prevenção, aborda aspectos sociológicos sobre as condições de trabalho frequentemente relacionadas com este tipo de adoecimento e categorias de trabalhadores mais acometidos. Focaliza aspectos médicos sobre a LER/DORT e as Síndromes de Bloqueio na MTC, estabelecendo canais de articulação e contribuição entre esses campos de conhecimento sobre a saúde humana. Ressalta o Qi Gong como importante prática terapêutica e preventiva na MTC e sua adequação à LER/DORT. No âmbito do Qi Gong, focaliza o Lian Gong Shi Ba Fa - Lian Gong em 18 Terapias, já amplamente experimentado na China e divulgado, inclusive no Brasil, para tratar e prevenir síndromes de dor em região cervical, ombros, membros superiores, membros inferiores e região lombar. À guisa de conclusão, são apresentadas algumas reflexões sobre a propriedade e a importância do Qi Gong na reabilitação e na prevenção da LER/DORT.

Palavras-chave: LER/DORT; saúde do trabalhador; medicina tradicional chinesa; Qi Gong.

\begin{abstract}
This paper evaluates Qi Gong - therapeutic exercises, one of the pillars of the Traditional Chinese Medicine (TCM) - as an important tool of preventing Cumulative Trauma Disorders (CTD) and healing, using allopathic medicine, TCM, and sociology of work elements. Considering its interdisciplinary nature and various levels of CTD prevention, this study examines the sociological aspects of work conditions that are usually associated with CTD and with groups that most frequently report symptoms. It focuses on CTD medical aspects and on TCM Blocking Syndromes, opening channels of communication and contribution between these fields of knowledge. It emphasizes that Qi Gong is an important therapeutic and preventive practice within TCM, and that is suitable for treating CTD. Within Qi Gong, it emphasizes Lian Gong Shi Ba Fa - Lian Gong in 18 therapies, which has been broadly experienced in China, and also publicized in Brazil, for treatment and prevention of pain syndromes in the upper and lower limbs, as well as in the cervical and lumbar regions. It finalizes by presenting considerations on the suitability and importance of Qi Gong for rehabilitation and prevention of CTD.
\end{abstract}

Keywords: cumulative trauma disorders; occupational health; traditional chinese medicine; Qi Gong. 


\section{Introdução}

O presente ensaio apresenta elementos da medicina ocidental, da medicina tradicional chinesa e da sociologia do trabalho, visando refletir sobre as possibilidades e os limites do Qi Gong ${ }^{4}$ para a prevenção da LER/DORT e para a recuperação da saúde.

Tais reflexões precisam divisar tanto as possibilidades, quanto os limites do uso do Qi Gong com estes objetivos. Isto porque a LER/DORT é uma forma de adoecimento fortemente dependente da organização e das condições de trabalho. A centralidade do trabalho na geração da LER/DORT é, hoje, reconhecida no campo científico internacional e nacional da saúde e trabalho. Portanto, a prevenção e a reabilitação da LER/DORT requerem contribuições interdisciplinares - da medicina clínica, da ergonomia, da psicodinâmica do trabalho e da sociologia do trabalho, dentre outras.

As ações preventivas e reabilitadoras precisam envolver conhecimentos em todos estes campos e fundamentar diferentes níveis de mudanças necessárias. Num primeiro nível, macrossocial, trata-se de mudanças para liberar o trabalho das amarras da ótica unilateral da produtividade cega e do lucro desmedido das empresas. São mudanças de caráter estrutural, profundas e de longo prazo nas sociedades atuais, nas quais ainda prevalecem as formas predatórias de trabalho alienado. Ações neste nível requerem mudanças profundas de consciência sobre o trabalho e a vida social e transformação do trabalho patogênico em trabalho digno e saudável.

Em segundo lugar, mudanças - de caráter coletivo, mas pontuais - na organização e nas condições de trabalho. Referimo-nos à fragmentação do trabalho, aos ritmos intensos, à pressão de tempo, às metas inalcançáveis, às jornadas de trabalho exaustivas, às necessidades de pausa, de descanso etc., aspectos relacionados aos limites biopsicossociais dos seres humanos que precisam ser respeitados pela gestão do trabalho. Mudanças neste nível têm sido implementadas em maior ou menor grau, em diferentes países, desde fins da década de 1960, passando por retrocessos desde os anos de 1990 com a crescente terceirização e precarização do trabalho.

Em terceiro lugar, referimo-nos às medidas preventivas envolvendo o fortalecimento dos indivíduos. Fortalecimento em dois sentidos interrelacionados, mas irredutíveis entre si. Num primeiro sentido, trata-se de fortalecer a consciência crítica e transformadora dos indivíduos sobre o mundo real do trabalho. Esta consciência é a base para as mudanças nas condições aviltantes de trabalho prevalecentes hoje. Num outro sentido, o foco é o fortalecimento do indivíduo en- quanto unidade orgânica mente/corpo, favorecendo a integração biopsíquica, a saúde e sua construção como cidadão ser humano.

Nesta dimensão, a medicina tradicional chinesa - e o Qi Gong especificamente - tem duas características importantes que justificam a necessidade de sua incorporação, difusão e popularização no ocidente. Por um lado, consiste numa prática milenar, ou seja, consagrada, experimentada, amplamente testada ao longo de muitas gerações humanas, constituindo, hoje, um patrimônio da humanidade voltado para a preservação da capacidade vital e da saúde. Por outro lado, o Qi Gong é uma prática terapêutica que pode ser compreendida, aprendida e exercida pelas pessoas com relativa facilidade, apesar de sua complexidade, rompendo com a passividade dos tratamentos ocidentais de saúde e com o acesso restrito às práticas preventivas e holísticas.

Objetivamos com este ensaio, trazer elementos para esta compreensão, elucidando que a prevenção da LER/ DORT precisa se dar em vários planos e que o Qi Gong pode ter um papel fundamental para trabalhar o plano do indivíduo. Pensamos ser indispensável a interação entre práticas de fortalecimento mente/corpo do indivíduo (Qi Gong) e atividades de formação crítica sobre o trabalho alienado contemporâneo para que se potencialize a capacidade de adaptação e de transformação dos indivíduos em sociedade e não apenas a adaptação individual a uma vida social patogênica.

A difusão e a popularização do Qi Gong como prática preventiva da LER/DORT e de recuperação da saúde pode se dar, dentre outras formas, através de: i) propostas integradas de formação teórica social crítica e prática de Qi Gong para populações de risco, contemplando tanto elementos teóricos da sociologia do trabalho, da psicologia social, da psicodinâmica do trabalho, da saúde do trabalhador, da ergonomia, dentre outros - que qualificam e permitem ressignificar o desgaste da saúde no trabalho à luz do conhecimento sobre as condições e a organização do trabalho patogênico, - quanto o fortalecimento dos indivíduos com a prática do Qi Gong; ii) estudos e pesquisas que contribuam para a sua inclusão em políticas públicas de saúde visando, sobretudo, à prevenção e à recuperação da saúde em indivíduos sob condições de trabalho que geram LER/DORT.

Inicia-se este ensaio com breves considerações sobre a conceituação e a prevalência das LER/DORT no mundo e no Brasil. Em seguida, são apresentados aspectos médicos sobre LER/DORT (formas clínicas, estágios de adoecimento e tratamento) e sobre a Síndrome Bi na Medicina Tradicional Chinesa (obstrução dolorosa que acomete frequentemente articulações, músculos e tendões). Em seguida, são abordados aspectos gerais sobre o Qi Gong, focalizando-se o Lian Gong Shi Ba Fa

\footnotetext{
${ }^{4}$ Qi Gong ou Chi Kung: literalmente “exercícios de energia”, consiste em práticas milenares usadas para estimular, nutrir e canalizar o fluxo de energia na rede de meridianos do organismo humano, revitalizando o corpo e a mente. Constitui um dos pilares da Medicina Chinesa, juntamente com a Fitoterapia/Farmacologia, Dietoterapia, Tui Ná e Acupuntura/Moxabustão.
} 
- Lian Gong em 18 Terapias, já amplamente experimentado e divulgado desde 1975, "para tratar e prevenir síndromes doloridas no pescoço, nos ombros, na região lombar e nas pernas" (ZHUANG, 2000, p. 6). À guisa de conclusão, são apresentadas algumas reflexões sobre a prevenção da LER/DORT e o Qi Gong.

\section{LER/DORT: conceituação e breve histórico}

A LER/DORT, ${ }^{5}$ hoje, é reconhecida legalmente no Brasil e em vários países como uma doença estreitamente relacionada ao trabalho.

O termo LER - Lesão por Esforços Repetitivos - foi adotado no Brasil, entre 1986 e 1998, equivalente aos termos utilizados na Austrália: OOS - Occupational Overuse Syndrome e posteriormente RSI - Repetition Strain Injuries. Nos EUA e demais países anglo-saxônicos: CTD - Cumulative Trauma Disorders, dentre outros. No Brasil, esta denominação foi alterada para DORT - Distúrbios Osteomusculares Relacionados ao Trabalho, através da OS nº 606, de 05 de agosto de 1998 do Instituto Nacional de Seguro Social (INSS) e, "em 2003, esse instituto alterou novamente a terminologia para LER/DORT”, conforme Almeida (2009, p. 25).

Constam da lista de doenças relacionadas ao trabalho do Ministério da Saúde e do Ministério da Previdência Social as seguintes entidades nosológicas, passíveis de serem reconhecidas como LER/DORT, conforme Maeno et al. (2006, p. 22-23):

Síndrome cervicobraquial; Dorsalgia; cervicalgia; Ciática; Lumbago com ciática; Sinovites e tenossinovites, Dedo em gatilho, Tenossinovite do estilóide radial (Tenossinovite de De Quervain); Outras sinovites e tenossinovites; Sinovites e tenossinovites não especificadas; Transtornos dos tecidos moles relacionados com o uso, o uso excessivo e a pressão, de origem ocupacional; Sinovite crepitante crônica da mão e do punho; Bursite da mão; Bursite do olecrano; Outras bursites do cotovelo; Outros transtornos dos tecidos moles relacionados com o uso, o uso excessivo e a pressão; Transtorno não especificado dos tecidos moles relacionados com o uso, o uso excessivo e a pressão; Fibromatose da fáscia palmar (contratura ou moléstia de Dupuytren); Lesões do ombro; Capsulite adesiva do ombro (ombro congelado, periartrite do ombro); Síndrome do manguito rotador ou síndrome do supra-espinhoso; Tendinite biciptal; Tendinite calcificante do ombro; Bursite do ombro; Outras lesões do ombro; Lesões do ombro não-especificadas; Outras entesopatias; Epicondilite medial; Epicondilite lateral (cotovelo de tenista), Outros transtornos especificados dos tecidos moles não classificados em outra parte (inclui mialgia).

Maeno et al. (2006, p. 25) destacam a síndrome do impacto como entidade nosológica frequente, porém "não especificada no Manual de Procedimentos para os Serviços de Saúde/Doenças Relacionadas ao Trabalho”. Salientam a importância, em conformidade com a literatura internacional, da síndrome do túnel do carpo, da síndrome do canal de Guyon, da síndrome do pronador redondo, da síndrome do desfiladeiro torácico, dentre outras. Chamam a atenção, ademais, para a Síndrome Dolorosa Miofascial (SMF), a Fibromialgia e a Síndrome Complexa de Dor Regional (SCDR), processos crônicos de difícil manejo que estão frequentemente relacionadas com LER/DORT. ${ }^{6}$

As discussões em torno da terminologia se estendem também à própria existência da patologia, à sua relação com o trabalho (riscos ocupacionais) ${ }^{7}$ e à conduta após o diagnóstico. Conforme Almeida (2009, p. 26), vale salientar seu reconhecimento por:

organismos internacionais de referência na área de Saúde e Segurança Ocupacional, com credibilidade no meio científico, como a Occupational Safety and Health Administration - OSHA e o National Institute of Occupational Safety and Health - NIOSH.

Apesar das controvérsias, a incidência de LER/DORT - dos distúrbios musculoesqueléticos - tem aumentado nos últimos anos tanto no Brasil (a partir das ocorrências registradas pelos serviços de saúde e centros de estudos em Saúde do Trabalhador), quanto nos demais países, segundo vários autores, respondendo por grande parte dos adoecimentos no mundo do trabalho.

\footnotetext{
${ }^{5} \mathrm{~A}$ LER/DORT se inscreve na terminologia mais ampla dos Distúrbios Musculoesqueléticos (DME). "Os DME decorrem do comprometimento inflamatório ou degenerativo de tendões, ligamentos, bursas, articulações, incluídas as intervertebrais discais, circulação, nervos periféricos e raízes nervosas, em diferentes segmentos corporais como a região do pescoço e ombro, cotovelos, antebraços, punhos, mãos, região lombar e segmentos dos membros inferiores. O principal sintoma é a dor que pode resultar em prejúzo funcional. Os estudos sobre os mecanismos fisiopatológicos dos DME apresentam de maneira consistente para cada tecido, considerando sua função e estrutura, os efeitos da repetitividade, da compressão, da carga sobre a microestrutura do tecido, sobre as características biomecânicas e a função biológica (Fordet et al., 2002; NRC \& IM, 2001; Kuorinka \& Forcier, 1995)." (FERNANDES, 2004, p. 12). Estudos sobre DME serão referidos sempre que elucidativos para os objetivos deste ensaio.

${ }^{6}$ Ver, também, Azambuja, Tschiedel e Kolinger (2004): estudo de casos de LER/DORT em trabalhadoras portadoras de SMF e SDRC, sob acompanhamento ambulatorial no CIAST/SUS (Centro Integrado de Atenção à Saúde do Trabalhador), Secretaria Municipal de Saúde de Porto Alegre.

7 “Embora alguns autores tenham se recusado a admitir a etiologia ocupacional dos DME, gerando polêmicas e controvérsias na década de 90 (Silverstein et al., 1996), revisões sobre o tema conduzidas ao longo de duas décadas chegam a conclusão sobre a associação entre esses distúrbios e demandas físicas como repetitividade, posturas inadequadas e força [...]. Demandas psicossociais também têm sido mencionadas como fatores de risco para DME [...]. As teorias correntes para explicar a relação entre as demandas psicossociais e os DME são aquelas que associam diretamente o estresse gerado por essas demandas ao aumento de atividade muscular e aquelas que admitem haver uma influência deste estresse sobre a percepção dos DME, ou seja, consideram que na presença de condições de trabalho estressoras, haveria uma reduzida habilidade dos trabalhadores para lidar com os sintomas, aumentando sua percepção. No caso da atividade muscular aumentada, uma via neuroendócrina, com elevação de epinefrina, neuroepinefrina e cortisol tem sido indicada como resposta às demandas psicossociais.” (FERNANDES, 2004, p. 12-13, grifos nossos)
} 
Nos últimos vinte anos, a literatura internacional tem mostrado consistentemente a importância dos DME (Distúrbios Musculoesqueléticos) enquanto problema de saúde pública. Somente nos Estados Unidos, os DME são responsáveis por 56\% de todas as doenças ocupacionais. Em 1992, o custo médio por caso de distúrbio musculoesquelético foi de US\$8.070,00 contra US\$824,00 para outras doenças, com um custo global de mais de US $\$ 4,04$ bilhões. Em 1996, o custo com assistência foi mais do que US $\$ 418$ bilhões e, contados os custos indiretos, estima-se um total de US\$1,256 trilhão. A evolução da ocorrência nos E.U.A. pode ser evidenciada através do número de casos registrados: em 1981 houve 22.600 casos, em 1994 foram registrados 332.000 , portanto, um aumento de 14 vezes, e no ano de 1998, foram registrados 650.000 novos casos de DME relacionados com o trabalho (Brasil, 2000; Melhorn, 1998). (FERNANDES, 2004, p. 14)

No Japão, desde 1958, e na Austrália, desde a década de 1970, têm sido crescentemente detectados casos de distúrbio cérvico-braquial em diversas categorias de trabalhadores, conforme Assunção (1995, p. 75). O panorama é semelhante na Europa, onde os distúrbios musculoesqueléticos se tornaram uma prioridade para instituições como a European Agency for Safety and Health at Work, que deflagrou campanha específica para sua prevenção em 2001.

Prevalências de 30\% e 40\% são registradas em trabalhadores na Holanda e na Bélgica, respectivamente. Na Itália, 60\% dos DME são reconhecidos como doença ocupacional. Neste país, os casos de compensação securitária apresentaram um incremento de 873 para 2.000 entre os anos de 1996 e 1999. Na França, os DME representavam 40\% (2.602 casos) das doenças ocupacionais em 1992 e passaram a 63\% (5.856 casos) no ano de 1996 (Buckle \& Devereux, 2002). [...] No Reino Unido, estima-se que 5,4 milhões de dias de trabalho são perdidos anualmente devido aos DME (de pescoço e membros superiores) relacionados com o trabalho, equivalendo a aproximadamente um mês de trabalho por caso. Estima-se que os custos com esses distúrbios foram da ordem de 1,25 bilhão de libras por ano (Buckle \& Devereux, 2002). (FERNANDES, 2004, p. 59-60)

Na Alemanha, os distúrbios musculoesqueléticos tornaram-se o principal grupo de doenças a causar afastamentos com licença médica nos anos de 1980, chegando a representar mais de $30 \%$ dos adoecimentos registrados no início dos anos de 1990 e $26 \%$ em 2006, como revelam Elkeles e Seligmann-Silva (2010). Numa análise contextualizada, os autores focalizam a trajetória dos DME na Alemanha e a expansão dos distúrbios mentais na perspectiva do processo de flexibilização e precarização do trabalho (e também da reunificação alemã). Ao analisarem o recuo de licenças e da duração dos afastamentos nos anos 1990 e 2000, dão visibilidade não apenas ao absenteísmo por adoecimento, mas também à questão do presenteísmo, ${ }^{8}$ que contribui para o agravamento e a cronificação dos problemas de saúde.

Em suma, as LER/DORT constituem um fenômeno universal e crescente no mundo do trabalho contemporâneo, embora seu surgimento não seja recente. Há remotas referências na literatura médica, tais como as contribuições de Ramazzini no século XVII e de Fritz De Quervain que, em 1891, descreveu a "entorse das lavadeiras", posteriormente nomeada "tenossinovite do polegar" ou "enfermidade de De Quervain". Contudo, é notória sua expansão desde a segunda metade do século XX, em todos os setores de atividade - indústria, serviços, comércio - que tenham acentuado o caráter fragmentado do trabalho e a requisição de movimentos rápidos, intensos, repetitivos, sob pressão de tempo, metas e posturas inadequadas. A adoção de novas tecnologias de base microeletrônica e/ou alternativas organizacionais, tais como as práticas japonesas, tem intensificado estas características do trabalho e seus danos à saúde, demonstradas por estudos de caso em diversos países.

No Brasil, foram observados casos de LER/DORT ${ }^{9} \mathrm{a}$ partir dos anos 1980, em várias localidades. Em 2002, passaram de 19 mil casos - 91\% dos registrados como doença do trabalho (FERNANDES, 2004) - para 20 mil em 2006 e 117,5 mil em 2008, conforme Elkeles e Seligmann-Silva (2010). O Nexo Técnico Epidemiológico Previdenciário ${ }^{10}$ permitiu avanços importantes dos registros no país, aumentando a visibilidade dos DME - LER/DORT e dorsalgias. Contudo, sua aplicação tem encontrado resistências por parte das empresas e dificuldades institucionais. A despeito dos avanços nos registros das doenças do trabalho, é necessário considerar, como afirmam Elkeles e Seligmann-Silva (2010),

\footnotetext{
8 "Presenteísmo - conforme Goetzel et al. 2004 - é entendido como o fenômeno que em tempos de globalização e de preocupação em manter o trabalho se caracteriza pelo fato de uma parte dos empregados estarem presentes ao trabalho, mesmo doentes." (ELKELES E SELIGMANN-SILVA, 2010, p. 10)

9 “No Brasil, em função da inexistência de um sistema de informação para morbidade ocupacional, não há dados sobre a ocorrência desses distúrbios, mesmo para os quadros que implicam incapacidade para o trabalho, o que dificulta uma avaliação mais acurada da magnitude do problema. Os dados da Previdência Social, além de se restringirem à população com vínculo formal de emprego, beneficiária do auxílio-acidentário, referem-se àqueles casos reconhecidos pelos peritos do Instituto Nacional de Seguridade Social (INSS) e registrados como distúrbios osteomusculares relacionados com o trabalho (DORT). Portanto, representam apenas uma parte das ocorrências.” (FERNANDES, 2004, p. 15)

${ }^{10}$ NTEP: "Norma que instaura novo método de avaliação da incapacidade, baseada em critérios epidemiológicos. O NTEP estabelece relação estatístico-epidemiológica entre o código da doença de acordo à CID - Classificação Internacional de Doença - e o setor de atividade CNAE - Classificação Nacional de Atividades Econômicas - com base na série histórica dos benefícios concedidos pelo INSS (2000-2004). Deste modo, é presumido como acidentário o benefício por incapacidade em que o atestado médico apresenta um código de doença que tenha a relação com o CNAE da empresa na qual o/a segurado/a possui vínculo de trabalho.” (ELKELES E SELIGMANN-SILVA, 2010, p. 22-23)
} 
a questão do presenteísmo que acentua a defasagem entre a prevalência registrada e a prevalência real.

A flexibilização e a precarização do trabalho têm engendrado novas relações sociais e formas de organização do trabalho - marcadas por forte coerção e pressão de tempo - que podem exacerbar, substancialmente, as demandas físicas e as demandas psicossociais favorecedoras dos DME - LER/DORT. Sobre os fatores de risco ocupacionais e não ocupacionais, convém resgatar a excelente síntese feita por Fernandes (2004):

Revisões sobre a associação entre distúrbios músculoesqueléticos (DME) e ocupação conduzidas ao longo de quase duas décadas indicam que existe associação entre esses distúrbios e demandas físicas como movimentos repetitivos, posturas anômalas, uso de força e vibração (Buckle \& Devereux, 2002; NRC \& IM, 2001; Muggleton et al., 1999, Bernard 1997; Kuorinka \& Forcier, 1995; Stock, 1991; Hagberg \& Wegman, 1987). Demandas psicossociais também têm sido identificadas como fatores de risco para DME (Huang et al., 2003; Bongers et al., 2002; Huang et al., 2002; Devereux et al., 2002; Westgaard, 2000; Bongers et al., 1993). Entre as demandas psicossociais, a alta demanda psicológica, em especial, o ritmo acelerado de trabalho é o fator de risco para DME em extremidades superiores mais citado na literatura especializada. O efeito dos fatores psicossociais sobre os DME tem sido referido como parcialmente ou completamente independente das demandas físicas (Bernard, 1997). Citados muitas vezes indistintamente dos fatores da organização do trabalho que representam aspectos estruturais do processo de trabalho, os fatores psicossociais podem ser mais bem entendidos como "qualidades do ambiente organizacional subjetivamente experimentadas" (Huang et al., 2002:299). Fatores não ocupacionais foram também associados com DME em extremidades superiores, mas os achados não são muito consistentes. A presença de obesidade está associada com DME em extremidades superiores distais, mas os resultados apresentaram limites, por não considerarem adequadamente os fatores ocupacionais (Buckle, 1987). Também para idade os achados não são muito consistentes. Poucas evidências epidemiológicas existem quanto à associação entre atividades físicas esportivas e DME, bem como quanto à presença de filhos menores, embora citados na literatura como fatores associados aos DME em extremidades superiores (Bjorksten et al., 2001). A associação entre DME e sexo feminino é controversa (de Zwart et al., 2001; Buckle, 1997; Silverstein et al., 1987). (FERNANDES, 2004, p. 85, grifos nossos)

A European Agency for Safety and Health at Work considera diferentes grupos de fatores no desenvolvimento dos DME - inclusive LER/DORT. Resumidamente, compreendem os fatores físicos: força (exercida no desempenho do trabalho); movimentos repetitivos; posturas inadequadas; posturas estáticas (permanência prolongada em pé, sentado, com braços elevados acima dos ombros etc.); pressão localizada (pelo contato físico com ferramentas, superfícies etc.); vibrações; frio ou calor excessivo; iluminação deficiente (podendo ocasionar acidentes); elevados níveis de ruído (levando à tensão corporal). Os fatores organizacionais e psicossociais: falta de controle sobre as tarefas desempenhadas, demandas/carga de trabalho; baixo nível de autonomia; baixos níveis de satisfação no trabalho; trabalho repetitivo, monótono, executado com rapidez; falta de apoio de colegas, supervisores e gerentes. $\mathrm{E}$ os fatores individuais: história médica pregressa; capacidade/condicionamento físico; idade; obesidade, tabagismo (OSHA, 2007).

Nesta perspectiva, é necessário considerar a importância da Instrução Normativa 98/2003 (BRASIL, 2003) que permite alinhar o país com os avanços internacionais para a prevenção e a reabilitação no mundo do trabalho:

\begin{abstract}
A Instrução Normativa 98 surgiu como instrumento legal voltado a viabilizar a aplicação de muitos dos novos conhecimentos que haviam sido construídos, pela conjugação de diferentes estudos e pesquisas realizados internacionalmente. Por exemplo, considerou a importância das abordagens multifatoriais preconizando sua aplicação às ações voltadas ao diagnóstico, tratamento, prevenção e reabilitação. Valorizou conhecimentos e aplicações da ergonomia situada. Integrou fundamentos e orientações referentes aos aspectos organizacionais, biomecânicos, psicossociais e preconizou atenção às singularidades sócio-culturais e às subjetividades dos indivíduos. Considerou ainda, que as ações levassem em conta a comorbidade e, de modo especial, a interface entre DORT e saúde mental. A IN 98 ofereceu avanços também ao instituir critérios periciais atualizados voltados a uma avaliação socialmente contextualizada da natureza e nexo dos agravos, dos graus de incapacidade e dos encaminhamentos indicados para a recuperação da saúde e retorno à atividade. (ELKELES; SELIGMANN-SILVA, 2010, p. 22-23)
\end{abstract}

\section{Aspectos médicos da LER/DORT}

De forma resumida e adaptada, estágios evolutivos, quadros clínicos e procedimentos terapêuticos - com base em Bahia (1996, p. 36), Miranda (1998, p. 74) e Brasil (2006) - são os seguintes: Grau I, refere-se ao indivíduo com sensação de peso e desconforto no membro afetado; com dor espontânea nos membros superiores ou escápula, sem irradiação nítida; com dor leve e fugaz, às vezes, em "pontadas" durante a jornada de trabalho, sem interferir na produtividade; melhora com o repouso; os sinais clínicos estão ausentes; bom prognóstico.

No Grau II, o indivíduo apresenta dor persistente e mais intensa, de modo intermitente, tolerável. Este quadro clínico não impede o desempenho da atividade profissional, embora possa ocorrer redução da produtividade nos períodos de exacerbação. Com dor localizada, pode haver irradiação e sensação de formigamento, calor e leves distúrbios de sensibilidade. Demora mais a se recuperar com o repouso e a dor pode se manifestar no desempenho de atividades domésticas. Os sinais continuam ausentes, mas podem apresentar nodulações, hipertonia e dor à palpação da massa muscular; prognóstico favorável. 
No Grau III, o indivíduo apresenta dor mais persistente, forte, com irradiação mais definida; frequentes paroxismos dolorosos mesmo fora do trabalho, especialmente à noite. Perda de força muscular; parestesias; queda da produtividade e, às vezes, impossibilidade de executar a função; o repouso reduz a intensidade da dor; dificuldade ou impossibilidade de executar trabalhos domésticos; sinais clínicos como edema, hipertonia muscular, alterações da sensibilidade principalmente nos paroxismos com palidez ou hiperemia e sudorese da mão; a mobilização/palpação do grupo muscular acometido provoca dor forte; quadros com comprometimento neurológico compressivo; a eletroneuromiografia pode estar alterada. O retorno à atividade produtiva é problemático; prognóstico reservado.

O Grau IV cursa com dor forte, contínua, por vezes insuportável e intenso sofrimento. Os movimentos agravam a dor; há paroxismos de dor mesmo com imobilização do membro. Perda de força e do controle dos movimentos; edema persistente. Podem surgir deformidades e atrofias, especialmente dos dedos. A capacidade de trabalho é anulada; a invalidez se caracteriza pela impossibilidade de executar um trabalho regular; as atividades domésticas e da vida diária são altamente prejudicadas. Evolui, frequentemente, com alterações psicológicas tais como quadro de depressão, ansiedade e angústia. Prognóstico sombrio.

O tratamento depende do estágio evolutivo da doença, compreendendo, em geral, procedimentos tais como repouso em vários graus, medidas ergonômicas, imobilização, medicação (analgésicos e anti-inflamatórios não esteróides; psicotrópicos), fisioterapia (eletrotermofototerapia, hidroterapia, massoterapia, cinesioterapia, dentre outros), acupuntura e outras terapias complementares, terapia ocupacional, psicoterapia individual e/ou em grupo, cirurgia (indicação controversa).

\section{Canais de articulação entre a Medicina Ocidental e a Medicina Tradicional Chi- nesa: ${ }^{11}$ Qi Gong e LER/DORT}

Na Medicina Ocidental, os processos fisiopatológicos da LER/DORT estão relacionados ao uso excessivo de estruturas neuromusculares, tendões e articulações, por repetição de movimentos ou sobrecarga no trabalho. A regularidade de atividades laborais dessa natureza conduz à fadiga muscular local e aos processos de in- flamação, levando ao consumo excessivo de nutrientes nas partes moles (músculos, fáscias, tendões, ligamentos etc.) e ao esgotamento desta nutrição, provocando desequilíbrio fisiológico que resultará em microlesões:

\begin{abstract}
Fadiga muscular local é uma diminuição da resposta de um músculo a um estímulo repetido. É uma resposta fisiológica normal do músculo e é caracterizada por uma redução na capacidade de produzir força pelo sistema neuromuscular associada a uma diminuição na amplitude de potenciais da unidade motora. A fadiga muscular pode ocorrer durante contrações musculares tanto dinâmicas quanto estáticas [...]. A diminuição da resposta do músculo é devido a uma combinação de fatores que incluem: a) distúrbios nos mecanismos contráteis do próprio músculo devido a uma diminuição nas reservas de energia, a oxigênio insuficiente e a um acúmulo de ácido lático; b) influências inibitórias (protetoras) do SNC; c) possivelmente uma diminuição na condução de impulsos na junção mioneural, particularmente nas fibras rápidas. (KISNER; COLBY, 1998, p. 58-59)
\end{abstract}

A persistência de qualquer processo de uso excessivo neuromuscular conduz à exaustão que resulta em deterioração temporária ou permanente da força muscular, seja em portadores de doenças neuromusculares prévias, seja em indivíduos sadios/hígidos. A continuidade deste processo leva à cronificação, com fibrose dos tecidos e perda de flexibilidade, perda da capacidade de contratilidade e relaxamento, reduzida perfusão e nutrição tecidual, culminando em perda de função.

São processos fisiopatológicos compatíves com as Síndromes de Bloqueio da Medicina Tradicional Chinesa (MTC), ou seja, as Síndromes Bi (Bi Zheng). ${ }^{12}$ São síndromes decorrentes do bloqueio ou da obstrução de meridianos e colaterais, de membros que podem aprofundar e afetar os órgãos internos (zang), abrangendo múltiplas doenças. Tais obstruções/bloqueios podem se dar pela ação de agentes patogênicos externos (vento, frio, calor, umidade) e traumatismos que bloqueiam os fluxos de Qi (energia vital) e sangue em diferentes localizações: tendões, punhos, músculos, pele, ossos, órgãos internos, conforme Ottino, Sohier e Wei-Guo (2002), a Associação Estatal de Medicina e Farmácia Tradicionais Chinesas - AEMFTC (2004) e Liu e Hong (2005).

Assim, é possível repensar tais síndromes no contexto atual do mundo do trabalho que absorve a maior parte do tempo acordado de viver de milhares de pessoas. Nestes ambientes, os indivíduos são expostos aos agentes físicos (vibração, frio, calor, umidade, ruído, radiações), aos agentes químicos, aos ergonômicos e à

\footnotetext{
${ }^{11}$ A Portaria no 971, de 3 de maio de 2006, do Ministério da Saúde, aprova a Política Nacional de Práticas Integrativas e Complementares (PNPIC) no Sistema Único de Saúde, abrangendo a Medicina Chinesa (Acupuntura e práticas corporais de promoção e recuperação da saúde e prevenção de doenças), a Homeopatia, a Fitoterapia e o Termalismo/Crenoterapia. Entre 13 e 15 de maio de 2008 foi realizado, em Brasília, o I Seminário Internacional de Práticas Integrativas e Complementares em Saúde, com a participação de representantes de cinco países e da OMS (BRASIL, 2006).

${ }^{12}$ A MTC distingue quatro modalidades de Síndrome Bi: Bi Migratório (Xing Bi), relacionada ao Vento, com artralgia ou dores articulares erráticas, a dor se desloca. Bi Doloroso (Tong Bi), associada ao Frio, o sítio da dor é fixo. Bi Fixo (Zhua Bi), umidade, apresenta formas extremas de bloqueio, com fortes dores nos membros. Bi Calor (Re Bi), calor, com acometimento das articulações e sinais de inflamação, edema e dor. Ver Hiria Ottino et al. (2002); Liu e Pai (2005).
} 
organização do trabalho (pressão de tempo; alta intensidade e gestos repetitivos; sobrecargas de trabalho; longas jornadas de trabalho com posturas inadequadas; coações, ameaças e punições que levam à autoaceleração; e sujeição às condições penosas de trabalho). No caso da LER/ DORT, os trabalhadores, em geral, silenciam sua fadiga e dores durante muito tempo, com medo do desemprego, de punições e estigmas sociais, o que favorece a cronificação do processo fisiopatológico (FRANCO, 2003).

A atual gestão do trabalho pelo medo permeia estas situações que, além dos fatores patogênicos externos, traumatismos e enfraquecimentos decorrentes de excesso de trabalho, promovem nos indivíduos os chamados fatores patogênicos internos da MTC - pela exacerbação das emoções de raiva, medo, ansiedade, preocupação, tristeza etc. - que cotidianamente devem ser silenciadas e contidas nos ambientes de trabalho. A cronificação destes estados leva às desorganizações fisiológicas e, provavelmente, aos distúrbios de saúde mental, cada vez mais frequentes no mundo contemporâneo, muitos dos quais acompanham a LER/DORT.

A base fisiopatológica da Síndrome Bi é o bloqueio dos fluxos de $\mathrm{Qi}^{13}$ e sangue nos meridianos, articulações e membros. A redução da circulação conduz à estagnação do Qi e estase sanguínea, levando aos quadros de dor, contratura muscular, limitações de movimentos cuja cronificação produz atrofia muscular e perda de função. ${ }^{14}$

Conectar as formas clínicas da LER/DORT, conforme a medicina ocidental, com a síndrome de bloqueio da MTC é importante pois amplia o leque de possibilidades terapêuticas ao alcance dos adoecidos e reforça a necessidade de se encarar com o máximo de seriedade o "calcanhar de Aquiles" da LER/DORT que é a prevenção. Esta ponte com a MTC reforça a necessidade de abordagens mais preventivas para que se possa, de fato, evitar um adoecimento que é evitável, na grande maioria dos casos, com medidas no plano da organização e das condições do trabalho e medidas para o fortalecimento dos indivíduos (alimentação adequada, exercícios terapêuticos, equilíbrio trabalho/descanso etc.).

A MTC pode contribuir muito para uma mudança de mentalidade quanto ao trabalho, pois preconiza a necessidade de se respeitar os biorritmos e os limites biopsíquicos (inerentes às teorias do Yin/Yang e dos Cinco Movimentos, aplicados e reconhecidos na fisio- logia humana). Trata-se de uma medicina cujos princípios afirmam a necessidade de se equilibrar atividade e repouso, evitando-se os excessos de trabalho físico e/ou mental, que caracterizam, hoje, o mundo do trabalho. Esses fundamentos - milenares - da MTC apontam para a necessidade de se rever, seriamente, a organização do trabalho contemporâneo, a exemplo da duração e intensidade das jornadas de trabalho que estão incompatíveis com o equilíbrio fisiológico da grande maioria dos trabalhadores, dentre outros aspectos. A mudança da mentalidade sobre o trabalho é fundamental para que se evite e se reduza o exército de lesionados jovens que temos hoje no Brasil, particularmente por LER/DORT.

Em segundo lugar, a tônica do tratamento da Síndrome Bi e dos quadros correlatos (periartrite escápuloumeral, cotovelo de tenista/epicondilite lateral umeral, cervicalgias etc.) pela MTC é a remoção da estase sanguínea e do bloqueio de canais, retirando a rigidez muscular, as dores etc., conforme Zhang (1990); Liu e Hong (2005). Vários recursos terapêuticos são utilizados: acupuntura, moxabustão, tui ná, fitoterapia e Qi Gong. O Qi Gong é um dos pilares da MTC com ação terapêutica e preventiva, de fácil assimilação, que pode ser aprendido e praticado pelos indivíduos, permitindo acrescentar, aos cuidados de especialistas, a autoterapia, que potencializa a eficácia da prevenção/tratamento e reduz recidivas. A combinação variada desses vários recursos se dá em função das necessidades de cada indivíduo.

A medicina chinesa parte do princípio de que tratar significa restituir ao indivíduo a sua capacidade inata de adaptação e transformação orgânica face aos estímulos externos e internos, buscando reestabelecer a sua força antipatogênica natural (FAP). Esta força, que se expressa em energia vital (qi), permite estar no mundo mantendo as relações entre micro e macrocosmo (indivíduo/meio ambiente) pelo equilíbrio dinâmico do Yin/Yang. Esse equilíbrio se reflete nos ciclos fisiológicos que se processam pela manutenção dos fluxos adequados de energia (qi), sangue e líquidos orgânicos no indivíduo. Tais fluxos se dão através de uma rede de meridianos e canais secundários (luo)..$^{15}$

O sistema de meridianos forma uma rede de conexões entre as partes mais superficiais do corpo e as mais profundas, representadas pelos órgãos internos. Ligando os órgãos entre si e com as extremidades, os meridianos regulam e mantêm o equilíbrio entre o ex-

\footnotetext{
${ }^{13}$ Qi ou chi: força vital bioeletromagnética (WONG, 2003; LAM, 1991).

${ }^{14}$ Estes processos ocorrem sob diversas formas clínicas, tais como as tenossinovites (inclusive tenossinovite dos extensores dos dedos e a tenossinovite de De Quervain), epicondilites, bursites, tendinite do supraespinhoso e tendinite biciptal, cistos sinoviais, dedo em gatilho, contratura ou moléstia de Dupuytren, compressão dos nervos periféricos, síndrome do túnel do carpo, síndrome do canal de Guyon, síndrome do pronador redondo, síndrome cérvicobraquial, síndrome do desfiladeiro torácico, síndrome da tensão do pescoço ou mialgia tensional, dentre outros. Em suma, naqueles quadros clínicos, anteriormente vistos, que configuram a LER/DORT. Sobre as abordagens de dor crônica no Ocidente, ver Lima (2005).

${ }^{15}$ Os trajetos desse sistema de fluxos e de comunicação corporal recebem denominações que variam entre diferentes autores (e traduções): meridianos e canais (Jing e Luo) e vasos (Mai). Os termos variam também quanto à classificação dos meridianos em: i) principais, regulares ou primários; ii) extraordinários, irregulares, curiosos ou vasos maravilhosos; iii) divergentes ou distintos. Para mais detalhes ver Auteroche, Solinas e Mainville (2000); He e Ne (2001); Ysao (2001); Martins e Garcia (2003); Associação Estatal de Medicina e Farmácia Tradicionais Chinesas - AEMFTC (2004).
} 
terior e o interior, ${ }^{16}$ entre a parte inferior e superior do corpo, entre direita e esquerda, entre região frontal e dorsal. Este sistema compreende 12 meridianos principais; 8 meridianos extraordinários; 12 meridianos divergentes; 12 meridianos tendinomusculares; canais colaterais ou de conexão que são 16 grandes colaterais, com seus ramos longitudinal e transversal, inumeráveis ramificações superficiais; e 12 zonas cutâneas na superfície.

Os órgãos internos ${ }^{17}$ Zang (de natureza Yin) compreendem os sistemas energéticos do pulmão, rim, fígado, coração, pericárdio/circulação, sexualidade e baço/pâncreas, que "essencialmente conservam o Qi”. Os órgãos Fu (de natureza Yang) compreendem o estômago, intestino grosso, bexiga, vesícula biliar; intestino delgado e o triplo aquecedor (San Jiao). Cada órgão zang (yin) está ligado a um órgão fu (yang), formando pares acoplados com diferentes polaridades. O sistema de meridianos mantém estes órgãos num todo orgânico, envolvendo desde a superfície ao interior do corpo, estabelecendo conexões com as diversas estruturas corpóreas, os tecidos, os órgãos dos sentidos, as expressões orgânicas e as emoções. A diferença de polaridades promove o movimento interno.

Resumidamente, com base em Ysao, Yamamura e Oliveira (1991, p. 94), são as seguintes as relações dos órgãos internos com a energia mental: o coração está relacionado à alegria, à ansiedade, ao Shen/consciência; o pulmão, à tristeza, angústia, aos reflexos e ao instinto; o fígado, à raiva, ódio, irritação, nervosismo, tensão e indecisão; o baço/pâncreas à atividade cerebral, ao raciocínio, ao pensamento, à reflexão, à preocupação, à memória, à vontade de pensar e à obsessão. Os rins estão relacionados à insegurança, ao autoritarismo, ao medo, ao temor, ao pânico, à vontade de decisão (vontade de viver). ${ }^{18}$

Assim, corpo/mente são uma unidade e os processos fisiológicos e patológicos são compreendidos na perspectiva do indivíduo enquanto ser integral:

Os processos fisiológicos estão intimamente relacionados com as atividades mentais e com as emoções. As atividades mentais e as emoções são funções do cérebro e são sua resposta para o mundo externo. Porém, a teoria dos órgãos zang-fu sustenta que as emoções humanas e as atividades mentais estão intimamente conectadas com as funções fisiológicas dos cinco órgãos zang. Portanto, a função normal do cérebro depende da harmonia de todos os órgãos zang. Além disso, os distúrbios emocionais e as atividades mentais anormais certamente afetam adversamente as atividades fisiológicas dos cinco órgãos zang. O Basic Questions ${ }^{19}$ declara: 'O coração, o pulmão, o fígado, o baço e o rim armazenam respectivamente vitalidade, coragem, alma, intenção e determinação. (ASSOCIAÇÃO ESTATAL DE MEDICINA E FARMÁCIA TRADICIONAIS CHINESAS - AEMFTC, 2004, p. 244-45)

Em suma, os órgãos internos produzem, armazenam e mantêm a essência, o qi, o sangue e os fluidos corporais sustentando a vida orgânica. A harmonia no funcionamento dos zang-fu é fundamental para a integridade do indivíduo e para a manutenção de suas relações com o meio ambiente interno - homeostase - e com o meio ambiente externo. São sistemas energéticos mediante os quais se mantém a força antipatogênica natural (FAP) e, portanto, a capacidade de transformação e de adaptação do indivíduo.

A Medicina Chinesa sustenta que, por um lado, normalmente existe um estado de equilíbrio relativo entre os órgãos internos e os tecidos e, por outro lado, entre o meio ambiente e o corpo humano. O equilíbrio não é estático; é um estado de constante autoajuste. Dessa maneira, as atividades fisiológicas normais do corpo são asseguradas.

Se o corpo estiver impossibilitado de se ajustar às condições de mudanças, o equilíbrio dinâmico se perde e ocorrerão os distúrbios. (ASSOCIAÇÃO ESTATAL DE MEDICINA E FARMÁCIA TRADICIONAIS CHINESAS - AEMFTC, 2004, p. 333, grifos nossos)

Estes distúrbios assinalam a perda de FAP que corresponde a um afastamento do indivíduo em relação à Natureza e a suas leis. O afastamento da Natureza (dos ciclos fisiológicos, dos biorritmos) resulta em desequilíbrios (fragilizando o indivíduo diante de fatores patogênicos) que podem se traduzir em sinais e sintomas, configurando o processo de adoecimento que, se persistente, pode cronificar determinando lesões orgânicas.

\footnotetext{
${ }^{16}$ Exterior refere-se à pele, aos músculos e aos cursos superficiais dos meridianos e dos colaterais (Jing Luo). Interior: órgãos internos.

17 "Zang-fu é um termo geral para se referir aos órgãos internos, sendo cada um deles classificado em três grupos de acordo com suas funções fisiológicas (...).” (ASSOCIAÇÃO ESTATAL DE MEDICINA E FARMÁCIA TRADICIONAIS CHINESAS - AEMFTC, 2004, p. 242-243). São classificados em órgãos zang, órgãos fu e órgãos extraordinários (cérebro, medula óssea, vasos sanguíneos, vesícula biliar e útero. É importante observar que: "A concepção da Medicina Chinesa sobre os órgãos é diferente daquela do Ocidente. É considerada sob três aspectos: o energético, o funcional e o orgânico. Estes dois últimos correspondem à Fisiologia, à Histologia e à Anatomia Patológica estudadas no Ocidente. (...) Os Órgãos representados pelo coração, pulmão, fígado, baço/pâncreas e os rins são estruturas essenciais do organismo, responsáveis pela formação, crescimento, desenvolvimento e manutenção do corpo físico e mental. Cada órgão que representa um dos Cinco Movimentos tem a função de comando sobre os tecidos, por ele canalizados, na sua formação, e sobre a Energia Mental por ele elaborada" (YSAO, YAMAMURA E OLIVEIRA, 1991, p. 93-94).

${ }^{18}$ Ysao, Yamamura e Oliveira (1991) apresentam tabela associando estas relações com as estruturas corpóreas. Para as relações entre os órgãos zangfu com as emoções: He e Ne (2001); Associação Estatal de Medicina e Farmácia Tradicionais Chinesas - AEMFTC (2004), Li et al. (2004). Campiglia (2004) discute, especificamente, os desequilíbrios da Mente segundo a MTC e os impactos sobre os canais e a função Zangfu.

${ }^{19}$ Trata-se do Basic Questions, primeira parte do livro clássico Princípios da Medicina Interna do Imperador Amarelo (BING, 2001), composta de 81 capítulos. Sobre as relações corpo/mente na dor crônica, na biomedicina ocidental, ver Lima (2005).
} 
Ademais, é necessário observar que:

(...), o estresse emocional pode causar uma perturbação do fluxo do qi e dos distúrbios do yin e do yang, do qi e do sangue, como também dos órgãos zang-fu; esses fatores levam à fraqueza do qi antipatogênico. Portanto, a Medicina Tradicional Chinesa enfatiza a importância de um estado mental equilibrado para fortalecer o qi antipatogênico e prevenir doenças. As funções fisiológicas também são influenciadas pelos estilos de vida. Os maus hábitos, como horário irregular, sobrecarga de trabalho ou ociosidade e dieta imprópria, afetam, todos, a condição do qi antipatogênico, resultando em doenças endêmicas e comuns. (ASSOCIAÇÃO ESTATAL DE MEDICINA E FARMÁCIA TRADICIONAIS CHINESAS - AEMFTC, p. 335, grifos nossos)

A condição mental está estreitamente relacionada com as emoções. ${ }^{20}$ As emoções são expressões necessárias e naturais do ser humano, bem como a sua alternância e mudanças em função da experiência de estar no mundo. Para a MTC, as atividades emocionais estão intimamente vinculadas ao funcionamento dos órgãos internos, dos fluxos, da quantidade e qualidade do qi e do sangue, sendo o qi essencial dos órgãos a base material das emoções. Por outro lado, as emoções e seus transtornos - exacerbação e/ou persistência, preponderância continuada de um tipo de emoção em detrimento de seu fluxo alternado e variado - têm grande influência sobre a fisiologia energética dos órgãos internos. Assim:

Por exemplo, boas condições de trabalho tornam uma pessoa alegre e resultam na harmonia do qi e do sangue. (...) más condições de trabalho causam frustração e podem conduzir à doença. (ASSOCIAÇÃO ESTATAL DE MEDICINA E FARMÁCIA TRADICIONAIS CHINESAS, 2004, p. 344)

As sociedades contemporâneas encerram inúmeras situações laborais, cujas formas de organização e condições de trabalho - com fatores de risco físicos, organizacionais, e psicossociais - ultrapassam os limites biopsíquicos dos seres humanos, lançando os indivíduos em processo diversos de adoecimento, dentre os quais os DME. Sua prevenção requer, necessariamente, como vimos, mudanças em diversos níveis na estruturação de trabalho. No presente ensaio, focalizamos o plano do indivíduo, ressaltando a importância do Qi Gong como uma das ferramentas importantes e acessíveis de saúde pública.

A prática de Qi Gong - exercícios terapêuticos chineses, um dos pilares da medicina chinesa - contribui para harmonizar a energia vital (qi), equilibrando corpo e mente, mantendo e/ou recuperando a capacidade de transformação e adaptação do organismo e fortalecendo o indivíduo. Os reequilíbrios se dão através dos exercícios de desbloqueio, circulação e captação de energia, trabalhando os canais/meridianos tendinomusculares, divergentes, canais principais, vasos maravilhosos e o metabolismo dos órgãos internos.

\section{Aspectos gerais sobre o Qi Gong}

O Qi Gong é o resultado de alguns milhares de anos de experiência do homem no desenvolvimento do uso da energia vital para vários propósitos definidos. Os antigos mestres desenvolveram a arte da energia para curar doenças, promover a saúde, a longevidade, melhorar as habilidades de luta, expandir a mente, alcançar níveis diferentes da consciência e atingir a espiritualidade. Essas artes de cultivo da energia desenvolveram--se separadamente, apesar de, às vezes, terem influenciado umas às outras e serem conhecidas por nomes diferentes. Porém, todas elas tinham em comum a prática de exercitar os fluxos de energia: o chi ou qi. Desde a década de 1950, essas artes ficaram conhecidas como Qi Gong, ${ }^{21}$ a arte da energia.

O Qi Gong foi desenvolvido nos primórdios da civilização, ao se descobrir possibilidades de trabalhar a respiração - que é uma forma de obter energia - de diferentes maneiras, para propósitos específicos. Esses conhecimentos foram adquiridos por um método de tentativas e erros, mas, aos poucos, se acumularam para formar um corpo de conhecimento maior.

Os registros chineses mostram que por volta de 2.700 a.C., o Qi Gong tinha se tornado um aspecto importante da medicina chinesa. O tipo mais antigo era provavelmente uma forma de dança meditativa que estimulava o equilíbrio da energia do corpo (WONG, 2003). É impossível precisar a época e o surgimento do Qi Gong devido à sua antiguidade, porém sabe-se que recebeu profunda influencia do Budismo e da filosofia Taoísta.

Assim, os exercícios foram classificados em quatro tipos de Qi Gong que, hoje, estão amalgamados. O Qi Gong religioso (Budista e Taoísta), o Qi Gong intelectual, o Qi Gong marcial (escola interna e externa) e o Qi Gong médico. Neste último, destaca-se a contribuição de Hua Tuo, importante médico entre 141-208, durante a Dinastia Han, que desenvolveu sequências para preservar e recuperar a capacidade vital dos indivíduos, com o objetivo de manter e desenvolver a saúde física e psíquica através da mobilização da essência e do Qi.

\footnotetext{
${ }^{20}$ Os sete fatores emocionais (qi qing) reconhecidos na Medicina Chinesa são a alegria excessiva, a raiva, a melancolia, a ansiedade, o pesar [tristeza], o terror e o susto. Sob condições normais, as emoções são reações aos estímulos externos que não causam doenças. Porém, se a frustração emocional for extremamente abrupta, intensa ou persistente, e exceder assim a capacidade normal de suportar do corpo humano, é capaz, então de produzir distúrbios funcionais dos órgãos zang-fu, por perturbar o equilíbrio harmonioso do qi e do sangue. Essas emoções, portanto, tornamse os fatores patogênicos, provocando doença e lesões orgânicas. Em patogênese, os fatores emocionais diferem dos seis fatores exógenos que atacam o corpo por meio da boca, do nariz ou dos poros; em vez disso, os fatores emocionais originam-se no interior do corpo e afetam diretamente os órgãos internos relacionados (ASSOCIAÇÃO ESTATAL DE MEDICINA E FARMÁCIA TRADICIONAIS CHINESAS - AEMFTC 2004, p. 343).

${ }^{21}$ Neste ensaio, utiliza-se a grafia do termo romanizado oficial, em Pinyin: Qi Gong.
} 
O qi ou chi, força vital eletromagnética, permeia todos os seres no universo, podendo se transformar nos aspectos mais sutis e mais densos dos organismos existentes, através das mútuas interações entre matéria e energia que a tudo compõem. Na medicina chinesa, as atividades fisiológicas são explicadas a partir do movimento e da contínua transformação do qi, cujas principais funções são: ativar e promover o crescimento e desenvolvimento do corpo humano, dos órgãos internos e dos meridianos; governar a circulação do Xue (sangue) e controlar a distribuição dos líquidos orgânicos; promover e ativar os processos de transformação metabólica do organismo, inclusive de transformação dos alimentos ingeridos e do ar inalado, para formar sangue e líquidos orgânicos; aquecer o corpo, mantendo a temperatura adequada; proteger o organismo das invasões de fatores patogênicos externos; manter a anatomia das estruturas orgânicas e dos órgãos internos.

Os indivíduos, imersos no meio ambiente - numa complexa rede de seres - captam, transformam e eliminam diferentes formas de essências e de qi (energia). $\mathrm{O}$ equilíbrio destas interações e trocas nos indivíduos requer uma movimentação harmoniosa de qi nas oito portas (Ba men): alto/baixo, interior/exterior, frente/trás (ventral/dorsal), direita e esquerda do corpo. A prática do Qi Gong favorece o equilíbrio e a manutenção do fluxo de qi nas oito portas, preservando a integridade biopsíquica.

As práticas de Qi Gong envolvem, em geral, quatro fases. A primeira fase, de Desbloqueio, libera os canais para melhor captar as energias, fortalecer a estrutura óssea e a medula. Na segunda fase, de Captação, se busca captar a energia exterior. A fase de Circulação, em que os movimentos favorecem a circulação da energia captada pelos canais, para limpar, nutrir e fortalecer os órgãos internos. A fase de Armazenamento, quando se reserva a energia captada e circulada nos centros energéticos, para usar quando necessário.

As principais sequências de Qi Gong difundidas atualmente são: Tai Ji Qi Gong Shi Ba Shi (Tai Ji Qi Gong de 18 Passos); Lian Gong Shi Ba Fa (Lian Gong em 18 Terapias), Yi Qi Gong, Ba Duan Jin (As Oito Peças do Brocado de Seda), Lo Han Shi Ba Shou (18 Mãos de Buda), DaYan (Qi Gong do Ganso Selvagem - forma tradicional), Wu Qin Shi (Jogo dos Cinco Animais de Hua Tuo - Tradicional) e o Liu Zi Jue (6 Sons de Cura).

\section{Lian Gong Shi Ba Fa}

Dentre as sequências de Qi Gong, vamos focalizar, brevemente, o Lian Gong Shi Ba Fa, ou Lian Gong em 18 Terapias, que nas próprias palavras do Dr. Zhuang Yuan Ming, seu criador:

(...) é uma técnica que une medicina terapêutica e cultura física (cultura física significa, para os chineses, o fortalecimento harmonioso do corpo, permitindo o pleno funcionamento e utilização dos músculos, tendões e ossos, diferente da ideia no Ocidente, de cultura física como aumento da massa muscular e modelagem física). Consiste de um conjunto de exercícios que visam a prevenção e o tratamento de dores no pescoço, ombros, cintura, pernas e também doenças crônicas. Esta técnica foi por mim projetada, fruto de constantes pesquisas das heranças culturais - a Medicina Tradicional Chinesa, antigos exercícios terapêuticos e as artes guerreiras tradicionais (Wu Shu) - e a reflexão sobre os resultados de sua aplicação no campo terapêutico, ao longo de mais de 40 anos de prática clínica. Durante a minha longa práxis de tratar das causas e sintomas de dores no corpo e doenças crônicas, fui constantemente criando e aperfeiçoando técnicas de massagem e manobras manuais, adicionando a elas os conhecimentos da moderna medicina atual. Sintetizando todas estas vivências, transformei-as em exercícios, os quais projetei passo a passo para serem realizados pelo próprio paciente. (LEE, 1997, p. 9)

Atuante na área de ortopedia do Hospital Dong Chang Roand, de Shanghai, Dr. Zhuang tem uma vasta experiência no tratamento de pacientes com síndromes dolorosas. Aprofundou-se cada vez mais em estudos e observações para encontrar meios de melhorar o sofrimento dos seus pacientes. Dedicando-se à pesquisa aliada à sua formação, na qual foi "discípulo do grande mestre Wang Zhi Ping, considerado um dos maiores nomes em artes marciais e ortopedia da MTC, com quem aprendeu as artes marciais e também as técnicas de tratamento de problemas ortopédicos" (ZHUANG, 2000, p. 17-18) - organizou uma série de exercícios sistematizados com base na pesquisa de práticas milenares, tais como o Dao In, Tu Na Gong (técnica de respiração), o Jogo dos Cinco Animais e os Exercícios dos Oito Brocados de Seda, desenvolvendo, por fim, o Lian Gong em 18 Terapias. Com a sistematização das séries de exercícios, Dr. Zhuang buscou simplificar certos movimentos preservando a essência das práticas tradicionais, facilitando o seu aprendizado e tornando o tratamento, até então passivo, também em autotratamento. Em função dos resultados eficazes dessas práticas, com impactos, inclusive, sobre a saúde pública, o Lian Gong é difundido em toda a China desde 1975.

Em 1980, o Comitê Nacional de Esportes e Educação Física, a Central Nacional dos Sindicatos e o Ministério da Saúde emitiram um comunicado conjunto confirmando os excelentes resultados obtidos com a prática do Lian Gong e colocando-o, juntamente com a ginástica veiculada pelo rádio e o Tai Ji Quan, como uma das três ginásticas a serem divulgadas e praticadas na China inteira. Ainda não foram feitas contagens oficiais, mas já se sabe que há, hoje, em Shangai cerca de 500 centros de orientação e prática do Lian Gong, com mais de 200 mil praticantes, e na China inteira mais de 2.000 centros, com mais de três milhões de praticantes. (ZHUANG, 2000, p. 19)

Sua difusão para outros países iniciou-se no Japão, em 1984, prosseguindo para o Sudeste asiático, Indonésia, Estados Unidos, França, Canadá, Austrália e Brasil, dentre outros.

São exercícios terapêuticos especificamente criados para a prevenção e o tratamento de dores no corpo, cujos distúrbios têm como elemento comum a obstrução/bloqueio de qi e do sangue, afetando as estruturas e sua fisiologia. Esses bloqueios decorrem de vários fatores, anteriormente vistos: 
Os fatores que influenciam no surgimento de dores no corpo podem ser externos, como: vento, frio, umidade e secura; e internos, como: emoções negativas (raiva, preocupação, tristeza, medo, euforia), além da má utilização do corpo em posturas inadequadas, sedentarismo, esforço excessivo ou lesões. Estes fatores provocam o 'retardamento do qi e a estagnação do sangue', que produz fenômenos como espasmos, aderências e contratura dos tecidos moles, encurtamento dos músculos, ligamentos e tendões, os quais resultam em dores e dificuldade de movimentação (LEE 1997, p. 14).

Podem ser observadas contraturas, aderências, sinais inflamatórios nas regiões doloridas nos braços, ombros, dorso, região cervical, cintura, glúteos etc. A prática regular do Lian Gong permite a movimentação adequada para liberar músculos, fáscias, ligamentos, tendões, melhorando e expandindo a amplitude de movimentação das articulações.

Os exercícios foram concebidos tendo como base as estruturas anatômicas e a fisiologia de cada região: pescoço, ombros, região dorsal, região lombar, glúteos e pernas, dentro de uma visão global de todo o organismo. Assim, a prática do Lian Gong favorece uma "movimentação global coordenada e harmoniosa”, podendo agir numa patologia localizada, restaurando, porém, o equilíbrio do organismo como um todo, bem como os fluxos de alto e baixo, de expansão e recolhimento, de entrada e saída de qi. Para tanto, os movimentos devem ser lentos e contínuos, evitando-se a movimentação rápida ou abrupta, para que "os tecidos moles, que sofrem de patologias (contraturas, aderências, espasmos etc.) possam se relaxar e se soltar gradativamente, permitindo que a movimentação da articulação alcance o seu limite máximo," conforme Lee (1997, p. 16), evitando traumas e desconfortos.

O qi se move de forma contínua, fluindo pelos tecidos, órgãos e meridianos, em quatro direções básicas: partindo de um centro, ele ascende, descende, entra e sai. Quando sua movimentação está harmoniosa, o corpo se mantém saudável (...). Portanto, o movimento do Qi é a chave para manter o equilíbrio das várias funções psicofisiológicas. (...) A força interna exercita e ordena os músculos, tendões e ossos e estes, por sua vez, não obstaculizam o fluxo do zhen qi (qi verdadeiro). Pelo contrário, colaboram para que o mesmo circule plenamente, abrindo os meridianos e potencializando ainda mais a força interna (nei jing). (...) Para cultivar a força interna, os movimentos devem ser realizados de forma lenta, contínua e homogênea, acompanhados de respiração natural e coordenada. Não é possível realizar esta qualidade de movimento com a força muscular. A força muscular é mecânica e depende dos músculos, tendões e ossos; não tem continuidade e o seu efeito termina quando o potencial dos músculos, tendões e ossos declinam. (...) A força interna (nei jing) flui incessantemente pelo corpo alimentada pelas fontes do zhen qi. Ela mostra o seu potencial quando requisitada por uma intenção (yi) que a direciona. A intenção depende de uma mente concentrada e um coração tranqui- lo, requisitos básicos para gerar a força interna que anima o movimento do corpo para a ação desejada. (LEE, 1997, p. 117-118)

A sincronicidade - sem forçar - entre movimento e respiração potencializa esta harmonização, que juntamente com a correta execução dos exercícios são requisitos para alcançar resultados eficazes, favorecendo a recuperação das funções fisiológicas localizadas e globais.

Dados estatísticos vindos de diferentes partes da China demonstram que muitos trabalhadores, que sofriam de dores no corpo, obtiveram bons resultados terapêuticos com a prática do Lian Gong Shi $B a \mathrm{Fa}$. Especialmente os que trabalham em posturas fixas ou sentados por muito tempo, ao praticarem perseverantemente o Lian Gong Shi Ba Fa uma ou duas vezes ao dia, passam a sentir seus músculos - antes fatigados - equilibrados e restaurados, e os músculos - pouco usados, - ativados, obtendo a harmonia entre movimento e repouso, equilíbrio e coordenação. (LEE, 1997, p. 16)

Resultados observados em experiências clínicas (ZHUANG, 2000) sobre o uso terapêutico e preventivo do Lian Gong em 18 terapias revelam sua eficácia na eliminação de contraturas musculares e na promoção do relaxamento dos tecidos moles em pacientes com DME. Tais conclusões estão fundamentadas na observação de três grupos. No primeiro grupo de 71 pessoas com dores no pescoço, nos ombros, na cintura e nas pernas, o principal tratamento utilizado foi o Tui-ná (1 sessão a cada 2 dias). Os casos mais graves foram tratados, também, com fitoterápicos de uso externo ou interno. Cada ciclo de tratamento teve a duração de duas semanas. Após dois a quatro ciclos, $91,4 \%$ dos pacientes apresentaram algum tipo de melhora: 7 casos com reversão completa do quadro; 15 casos com melhora expressiva ("eliminação completa da dor espontânea, recuperação parcial da função motora; recuperação parcial da capacidade física anterior") e 43 casos (60,5\%) com melhora do quadro ("redução da dor espontânea, melhora da função motora, capacitação para atividades leves”). Em dois casos não ocorreu alteração do quadro e em quatro casos houve interrupção do tratamento (ZHUANG, 2000, p. 142).

No segundo grupo, formado por 217 pessoas com dores no pescoço, nos ombros, na cintura e nas pernas, o tratamento envolveu: Tui-ná nas regiões doloridas (1 sessão a cada 2 dias) e orientação para realizar o Lian Gong em 18 terapias, em casa, durante 30 minutos, duas vezes ao dia. Com um ciclo de tratamento de duas semanas, após dois a quatro ciclos observou-se que 96,6\% dos 217 casos apresentaram algum tipo de melhora $(22,5 \%$ com reversão completa; $27,1 \%$ com melhora expressiva e $47 \%$ com melhora do quadro). Sete casos $(3,2 \%)$ não apresentaram alteração. (ZHUANG, 2000, p. 143).

O terceiro grupo, formado por 1.361 pessoas com dores no pescoço, nos ombros, na cintura e nas pernas, foi tratado apenas com o Lian Gong em 18 terapias, duas vezes ao dia, durante 30 minutos. As pessoas foram treinadas em dez centros de orientação em Shan- 
ghai, realizando o Lian Gong duas vezes por dia, em sessões de meia hora. Novos exames realizados após dois ou quatro meses de tratamento revelaram que 98,2\% tiveram algum tipo de melhora, correspondendo a 1.361 pessoas $(38,4 \%$ com melhora expressiva e $59,8 \%$ com melhora do quadro) e $1,76 \%$ sem resultado. Ademais, resultados significativos foram obtidos em pesquisas com eletromiografia, demonstrando a redução/eliminação dos níveis de contraturas musculares e a obtenção de relaxamento dos tecidos moles (ZHUANG, 2000, p. 143-144).

Acrescentar o Lian Gong ao tratamento (fisioterapia, massagem, acupuntura, medicamentos, dentre outros), traz as seguintes vantagens, sintetizadas por Lee (1997, p. 15): aumento do poder imunológico do corpo, fortalecimento do físico, aumento do efeito terapêutico do tratamento médico, diminuição do tempo de tratamento, consolidação do efeito terapêutico, após o tratamento e prevenção de recaída da doença, além de facilitar o autocuidado para os pacientes que têm dificuldades de acesso aos médicos.

\section{Considerações finais: reflexões sobre a prevenção da LER/DORT e o Qi Gong}

Este ensaio traz elementos para refletirmos sobre as possíveis contribuições do Qi Gong para a prevenção da LER/DORT e a recuperação da saúde, à luz de conhecimentos da Medicina Ocidental e da Medicina Tradicional Chinesa. Apesar das diferentes perspectivas - quanto à etiologia, fisiopatologia, tratamento etc. - são abordagens que podem ser articuladas potencializando as possibilidades preventivas e terapêuticas para milhares de indivíduos com distúrbios e/ou lesões extremamente dolorosos e devastadores para suas vidas.

Esses canais de diálogo - tendo como foco a Síndrome Bi e a LER/DORT - precisam ser desenvolvidos e ampliados, com o aprofundamento nos campos da fisiopatologia ocidental e da fisiopatologia energética. Tarefa que requer estudos, pesquisas interdisciplinares e o esforço conjunto de vários pesquisadores.

O ensaio focaliza o Qi Gong para a prevenção da LER/DORT e a reabilitação, evidenciando seu poder de ação sobre o desbloqueio e a mobilização dos fluxos de qi, de sangue e de líquidos orgânicos no indivíduo, de forma harmoniosa e suave. Bloqueios nestes fluxos e circulação são a base para divisarmos a LER/DORT à luz dos fundamentos da medicina chinesa e da adequação do Qi Gong como prática preventiva e terapêutica.

Salienta-se a importância do Qi Gong na prevenção e no tratamento, não como recurso único ou excludente em relação aos demais. Como vimos, políticas preventivas e reabilitadoras em relação às LER/DORT precisam incluir dimensões sociais mais amplas, tais como: i) mudanças de mentalidade sobre o trabalho, sobre o modo de trabalhar e viver, no sentido de reestabelecer a relação Homem/Natureza, respeitando a fisiologia e os biorritmos dos indivíduos; ii) mudanças na gestão do trabalho - das condições e da organização do trabalho, - que veiculam tanto fatores patogênicos externos, quanto favorecem o desequilíbrio de fatores internos, quanto traumatismos e lesões/enfraquecimentos por excesso de trabalho; iii) no plano do indivíduo, fortalecer seu corpo e mente, favorecer a integração corpo/mente, nutrir sua força (interna) para ações coletivas, de mudanças sociais coerentes com a tríade primordial da relação natural Céu - Homem - Terra, que permite a todo ser humano se reconhecer enquanto um ser da espécie, um ser integrante da Natureza (FRANCO, 2003).

Assim, os problemas de saúde relacionados ao trabalho - LER/DORT, dentre outros, não podem ser melhorados/resolvidos apenas com prevenção e tratamento médico, que, via de regra, não cura os indivíduos. É necessário adotar, simultaneamente, perspectivas terapêuticas que fortaleçam os indivíduos - recuperando sua capacidade vital - e realizar mudanças na estrutura patogênica do trabalho contemporâneo. Os indivíduos e os sistemas sociais estão doentes.

Por fim, vale ressaltar a importância do Qi Gong como uma alternativa accessível, que pode ser apreendida e exercida pelos indivíduos. Apesar das distâncias culturais existentes, o caminho do Qi Gong, dentre outros, pode contribuir para romper o círculo vicioso dos tratamentos e recaídas, sendo, hoje, um dos tratamentos terapêuticos complementares reconhecido e preconizado pela Organização Mundial de Saúde (OMS). Portanto, é necessário difundir o Qi Gong, bem como traduzi-lo em linguagens compreensíveis, tanto pelos profissionais de saúde e gestores, quanto por pessoas leigas que não têm fácil acesso às práticas corporais holísticas dadas as barreiras socioculturais e econômicas.

\section{Referências}

ALMEIDA, A. C. E agora, o que será da minha vida? Estudo sobre os significados das LER atribuídos por operadores de telemarketing. 2009. 118 f. Dissertação (Mestrado em Saúde, Ambiente e Trabalho)-Faculdade de Medicina da Bahia, Universidade Federal da Bahia, Salvador, 2009.

ASSOCIAÇÃO ESTATAL DE MEDICINA E FARMÁCIA TRADICIONAIS CHINESAS - AEMFTC. Farmacologia e medicina tradicionais chinesas. São Paulo: Roca, 2004.
ASSUNÇÃO, A. A. Sistema músculo-esquelético: lesões por esforços repetitivos (LER). In: MENDES, R. (Org.). Patologia do trabalho. Rio de Janeiro: Atheneu, 1995. p. 173-212.

AUTEROCHE, B.; SOLINAS, H.; MAINVILLE, L. Atlas de acupuntura chinesa: meridianos e colaterais. São Paulo: Andrei, 2000.

AZAMBUJA, M. I. R; TSCHIEDEL, P. S. N.; KOLINGER, M. D. D. Síndrome miofascial e síndrome de dor 
regional complexa em pacientes com LER/DORT atendidos em ambulatório de saúde do trabalhador do SUS - identificação e caracterização clínica dos casos. Revista Brasileira de Medicina do Trabalho, Belo Horizonte, v. 2, n. 3, p. 176-184, jul./set. 2004.

BAHIA. Secretaria de Saúde do Estado da Bahia. Departamento de Vigilância da Saúde. Centro de Estudos de Saúde do Trabalhador. Manual de normas e procedimentos técnicos para a vigilância de saúde do trabalhador. Salvador: EGBA, 1996.

BING, W. Princípios da medicina interna do imperador amarelo. São Paulo: Ícone, 2001.

BRASIL. Instrução Normativa INSS/DC, № 98 de 5 de dezembro de 2003. Diário oficial [da] República Federativa do Brasil, Brasília, DF, 10 dez. 2003. Disponível em: <http://www81.dataprev.gov.br/sislex/ imagens/paginas/38/inss-dc/2003/anexos/IN-DC-98ANEXO. htm>. Acesso em: 30 jan. 2010.

. Portaria № 971 de 3 de maio de 2006.

Disponível em: <www.mpas.gov.br>. Acesso em: 30 jan. 2010.

CAMPIGLIA, H. Psique e medicina tradicional chinesa. São Paulo: Roca, 2004.

ELKELES, T.; SELIGMANN-SILVA, E. Trajetórias recentes dos distúrbios osteomusculares em dois contextos nacionais - Brasil e Alemanha. In: GLINA, D.; ROCHA, L. (Org.). Saúde mental no trabalho. Da teoria à prática. São Paulo: Roca, 2010 (no prelo).

FERNANDES, R. Distúrbios músculo-esqueléticos e trabalho industrial. 2004. 287 f. Tese (Doutorado em Saúde Coletiva)-Instituto de Saúde Coletiva, Universidade Federal da Bahia, Salvador, 2004.

FRANCO, T. Trabalho alienado: habitus \& danos à saúde humana e ambientais (O trabalho entre o céu, a terra e a história). 2003, 254 f. Tese (Doutorado)Faculdade de Filosofia e Ciências Humanas, Universidade Federal da Bahia, Salvador, 2003.

HE, Y. H.; NE, Z. B. Teoria básica da medicina tradicional chinesa. São Paulo: Atheneu, 2001.

KISNER, C.; COLBY, L. A. Exercícios terapêuticos: fundamentos e técnicas. 3. ed. São Paulo: Manole, 1998.

LAM, K. C. O caminho da energia. São Paulo: Manole, 1991.
LEE, M. L. Lian Gong em 18 terapias, forjando um corpo saudável: ginástica chinesa do Dr. Zhuang Yuan Ming. São Paulo: Pensamento, 1997.

LI, S. M. et al. Acupuntura e medicina tradicional chinesa. Florianópolis: IPE/MTC, 2004.

LIMA, M. A. G. Clínica da dor: sentidos e práticas no cotidiano dos espaços terapêuticos. 2005. 185 f. Tese (Doutorado)-Instituto de Saúde Coletiva. Universidade Federal da Bahia, Salvador, 2005.

LIU, G. W.; HONG, J. P. Tratado contemporâneo de acupuntura e moxibustão. São Paulo: Ceimec, 2005.

MAENO, M. et al. Lesões por esforços repetitivos (LER), distúrbios osteomusculares relacionados ao trabalho (DORT), dor relacionada ao trabalho: protocolos de atenção integral à Saúde do trabalhador de complexidade diferenciada. Brasília: Ministério da Saúde, 2006. Disponível em: <http://bvsms.saude.gov. br/bvs/publicacoes/protocolo_ler_dort.pdf $>$. Acesso em: 16 dez. 2006.

MARTINS, E. I. S.; GARCÍA, E. G. Pontos de acupuntura: guia ilustrado de referência. São Paulo: Roca, 2003.

MIRANDA, C. R. Introdução à saúde no trabalho. São Paulo: Atheneu, 1998.

OSHA. Factes 71. Disponível em: <http://ew2007. osha.europa.eu>. Acesso em: 31 jan. 2010.

OTTINO, H.; SOHIER, A.; WEI-GUO, H. Médecine chinoise: description de cent affections. Paris: Guy Trédaniel, 2002.

WONG, K. K. El gran libro de la medicina china. 3. ed. Barcelona: Urano, 2003.

YSAO, Y. Acupuntura tradicional: a arte de inserir. 2. ed. São Paulo: Roca, 2001.

YSAO, Y.; YAMAMURA, M. J. S.; OLIVEIRA, D. de S. Introdução ao Tai Chi Chuan, Tui Ná e Tao Yin. São Paulo: Centro de Pesquisa e Estudo da Medicina Chinesa, 1991.

ZHANG, E. Clinic of traditional chinese medicine II. Shanghai: Publishing House of Shanghai University of Traditional Chinese Medecine, 1990.

ZHUANG, Y. M. Lian Gong Shi Ba Fa. São Paulo: Pensamento, 2000. 